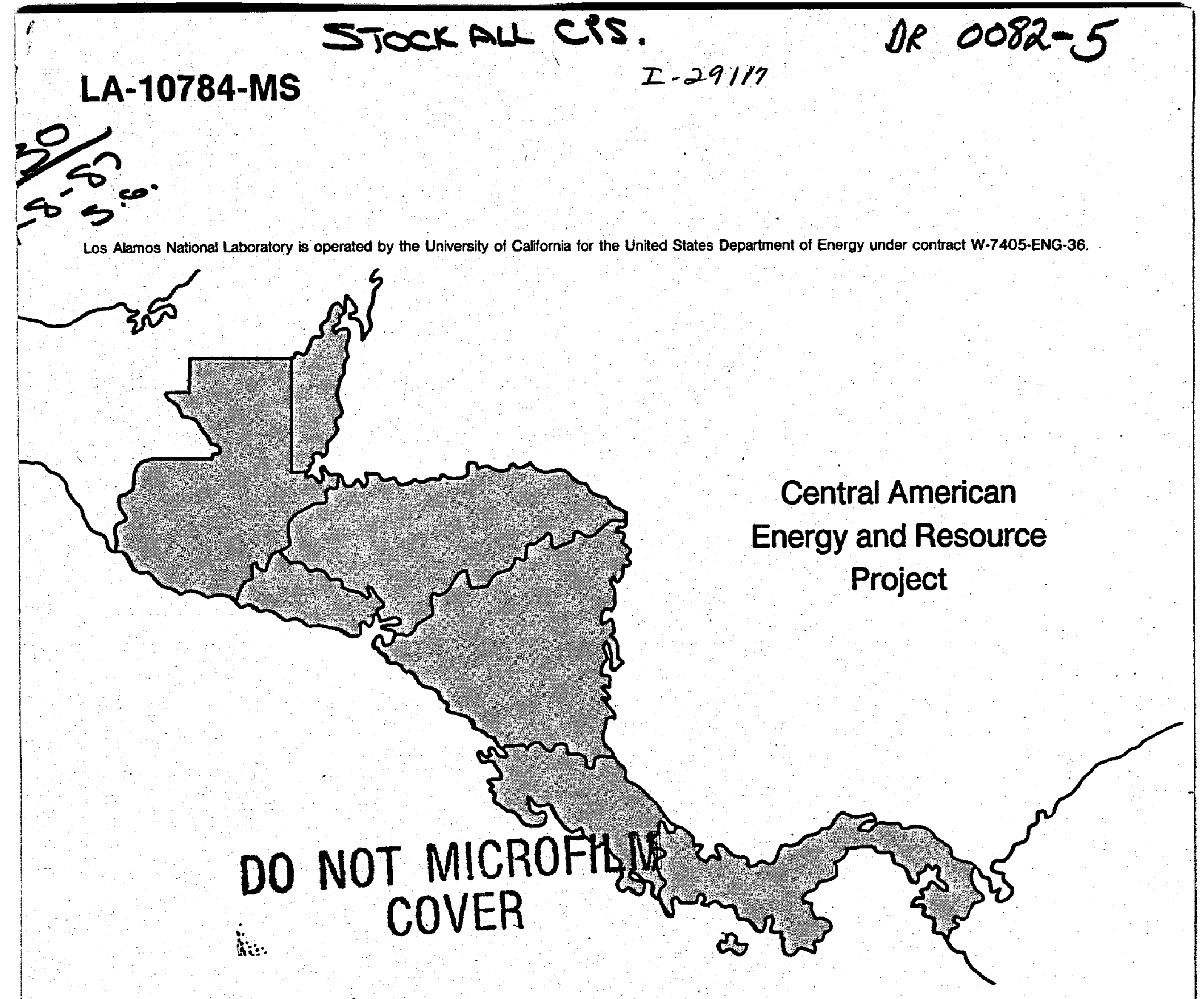

\title{
Catalog of Known Hot Springs and Thermal Place Names for Honduras
}

\section{MASTER}

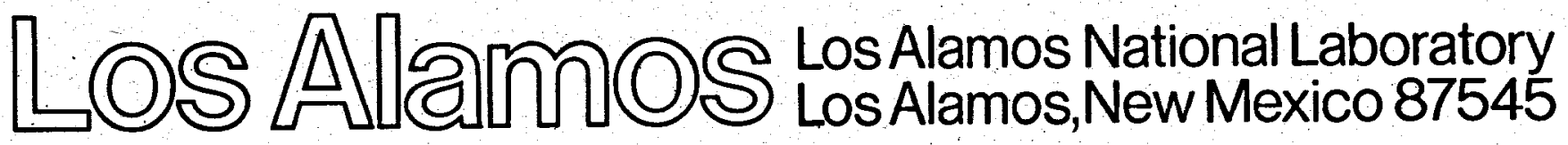




\section{DISCLAIMER}

This report was prepared as an account of work sponsored by an agency of the United States Government. Neither the United States Government nor any agency Thereof, nor any of their employees, makes any warranty, express or implied, or assumes any legal liability or responsibility for the accuracy, completeness, or usefulness of any information, apparatus, product, or process disclosed, or represents that its use would not infringe privately owned rights. Reference herein to any specific commercial product, process, or service by trade name, trademark, manufacturer, or otherwise does not necessarily constitute or imply its endorsement, recommendation, or favoring by the United States Government or any agency thereof. The views and opinions of authors expressed herein do not necessarily state or reflect those of the United States Government or any agency thereof. 


\section{DISCLAIMER}

Portions of this document may be illegible in electronic image products. Images are produced from the best available original document. 
This work was supported by the U.S. Agency for International Development.

\section{DO NOT MICROFILM $\because$ COVER}

This report was prepared as an account of work sponsored by an agency of the United States Government. Neither the United States Government nor any agency thereof, nor any of their employees, makes any warranty, express or implied, or assumes any legal liability or responsibility for the accuracy, completeness, or usefulness of any information, apparatus, product, or process disclosed, or represents that its use would not infringe privately owned rights. Reference herein to any specific commercial product, process, or service by trade name, trademark, manufacturer, or otherwise, does not necessarily constitute or imply its endorsement, recommendation, or favoring by the United States Government or any agency thereof. The views and opinions of authors expressed herein do not necessarily state or reflect those of the United States Government or any agency thereof. 


\title{
Catalog of Known Hot Springs and Thermal Place Names for Honduras
}

\author{
R. C. Finch*
}

\section{DISCLAIMER}

This report was prepared as an account of work sponsored by an agency of the United States Government. Neither the United States Government nor any agency thereof, nor any of their employees, makes any warranty, express or implied, or assumes any legal liability or responsibility for the accuracy, completeness, or usefulness of any information, apparatus, product, or process disclosed, or represents that its use would not infringe privately owned rights. Reference herein to any specific commercial product, process, or service by trade name, trademark, manufacturer, or otherwise does not necessarily constitute or imply its endorsement, recommendation, or favoring by the United States Government or any agency thereof. The views and opinions of authors expressed herein do not necessarily state or reflect those of the United States Government or any agency thereof.

\section{MASTER}

"Collaborator at Los Alamos. Department of Earth Sciences, Tennessee Technological University, Cookeville, TN 38505. 
CATALOG OF KNOWN HOT SPRINGS AND THERMAL PLACE NAMES FOR HONOURAS

$$
\text { by }
$$

R. C. Finch

\section{ABSTRACT}

Thermal place names were compiled from all 1:50,000 topographic quadrangle maps for the Republic of Honduras as of July 1986, from other Dublished maps, and from several sources of unpublished data. Known hot spring sites include those visited by Empresa Nacional de Energía Eléctrica (Honduras) geologists, sites visited by Los Alamos geologists in 1985, and other sites known to R. C. Finch. The number of known hot spring sites in Honduras with temperatures $>30^{\circ} \mathrm{C}$ is 125 . In addition, 56 thermal sites are suspected on the basis of thermal place names. The total number of geothermal sites, known and suspected, is 181. 


\section{INTRODUCTION}

Thermal place names were compiled from all 1:50,000 topographic quadrangle maps for the Republic of Honduras as of July 1986, from other published maps, and from several sources of unpublished data. Known hot spring sites include those visited by Gislason and Empresa Nacional de Energía Eléctrica (ENEE) geologists (Gislason, 1980), sites visited by Los Alamos geologists in 1985, and other sites known to R. C. Finch.

This list was compiled in March 1986 by Finch and by students working under his direction. The number of known hot spring sites in Honduras with temperatures $>30^{\circ} \mathrm{C}$ is 125 . In addition, 56 thermal sites are suspected on the basis of thermal place names. The total number of geothermal sites, both known and suspected, is 181 .

\section{METHOD OF COMPILATION OF HOT SPRINGS LISTED}

Sites listed as known hot springs include the following: "(1) Sites reported by Gislason (1980) were listed. The sites reported by Gislason were examined on the 1:50,000 topographic sheets to see if thermal place names were associated with the reported hot springs, and Gislason's site coordinates were checked against any other available data. In a number of cases Gislason's sites had been visited by Finch or members of the LoS Alamos team. Where Finch's or the Los Alamos team's locations disagreed with Gislason's coordinates, Finch's were used. (2) Sites not reported by Gislason but known to Finch from previous work in Honduras were listed, as were any sites not listed by Gislason but visited by the LOS AlamoS-ENEE team le.g., the Sambo Creek and Azacualpa sites). (3) Sites not listed by Gislason but reported by other authors considered reliable were, listed le.g., reports by Helbig, 1959, and Young, 1847). (4) Reliable verbal reports from scientists (e.g., K. Hirth, archaeologist at the University of Kentuckyl were listed. Sites listed as suspected thermal sites are those with geothermal place names found on the $1: 50,000$ topographic sheets, but with the thermal spring not verified by a visit. 
Maps covering the entire Republic of Honduras were examined. Finch and a group of students under his supervision examined the maps, searching for the following thermal place names:

(1) agua caliente,

(2) quebrada agua caliente (or any of several variants of this name) ;

(3) hervideros,

(4) agua tibia,

(5) fuentes termales, and

(6) agua termal (or any variant of this name).

They reported all thermal place names, along with their sites, using the UTM grid method. All names and site coordinates reported by students were verified by Finch and crosschecked with other geothermal site reports to avoid duplication of site listings. The author checked the accuracy and thoroughness of the students' data by comparing their list against the Gislason list of spring sites. 
III. HOT SPRING LISTING

\begin{tabular}{|c|c|c|c|c|c|c|}
\hline quadrangle Name & $\begin{array}{l}\text { UTM Grid } \\
\text { Coordinates }\end{array}$ & $\begin{array}{l}\text { Hot } \\
\text { Spring }\end{array}$ & $\begin{array}{l}\text { Place } \\
\text { Name }\end{array}$ & Place Name Identifier & Temperature & Comments \\
\hline \multirow[t]{2}{*}{ ARAMECINA } & $44 / 11$ & & $x$ & Agua Caliente & & \\
\hline & $45 / 14$ & & $x$ & Agua Cariente & & \\
\hline ARENAL & $100 / 051 *$ & $x$ & $x$ & $\begin{array}{l}\text { Agua Caliente } \\
\text { quebrada del Agua } \\
\text { Caliente }\end{array}$ & $61^{\circ}-67^{\circ} \mathrm{C}$ & $\begin{array}{l}\text { Located in the Aguán Valley, } \\
\text { presumably related to the Aguan } \\
\text { fault system. }\end{array}$ \\
\hline \multirow[t]{2}{*}{$\begin{array}{l}\text { AZACUALPA- } \\
\text { RIOO GUAYAMBRE }\end{array}$} & $99 / 00$ & & $x$ & $\begin{array}{l}\text { El Agua Caliente } \\
\text { Quebrada Agua Caliente }\end{array}$ & & $\begin{array}{l}\text { Probably associated wi th Río } \\
\text { Guayape fault system. }\end{array}$ \\
\hline & $00 / 99$ & $x$ & $x$ & $\begin{array}{l}\text { Quebrada El Agua } \\
\text { Caliente }\end{array}$ & & $\begin{array}{l}\text { Warm spring, probably associated } \\
\text { with Guayape fault system. }\end{array}$ \\
\hline \multirow[t]{2}{*}{ BAHIA CHISMUYO } & $394 / 911$ & $x$ & & & $45^{\circ} \mathrm{C}$ & \\
\hline & $433 / 918$ & $x$ & & & $38^{\circ} \mathrm{C}$ & \\
\hline BALFATE & $759 / 749 \star$ & $x$ & $x$ & $\begin{array}{l}\text { Quebrada Agua } \\
\text { Caliente. }\end{array}$ & $59^{\circ} \mathrm{C}$ & $\begin{array}{l}\text { Possibly associated with } \\
\text { Cordillera Nombre de vios } \\
\text { frontal fault. }\end{array}$ \\
\hline \multirow[t]{3}{*}{, } & $645 / 417 \star$ & $x$ & $x$ & $\begin{array}{l}\text { Agua Callente and } \\
\text { Aguas Termales }\end{array}$ & $59^{\circ} \mathrm{C}$ & $\begin{array}{l}\text { Possibly associated with } \\
\text { Cordillera Nombre de Dios } \\
\text { frontal fault; springs issue } \\
\text { from Paleozoic metamorphic rocks } \\
\text { (Pm). }\end{array}$ \\
\hline & $684 / 412$ & $x$ & & & $36^{\circ}-55^{\circ} \mathrm{C}$ & Hot springs issue from Pm. \\
\hline & $759 / 449$ & $x$ & & & $44^{\circ} \mathrm{C}$ & . \\
\hline \multirow[t]{2}{*}{ BARACOA } & $001 / 408 *$ & $x$ & $x$ & $\begin{array}{l}\text { Río Agua Caliente } \\
\text { and Agua Caliente }\end{array}$ & $58^{\circ}-68^{\circ} \mathrm{C}$ & $\because$ \\
\hline & $118 / 414$ & $x$ & & & $31^{\circ}-38^{\circ} \mathrm{C}$ & \\
\hline \multirow[t]{2}{*}{ CAMPAMERTO } & $45 / 15$ & & $x$ & $\begin{array}{l}\text { Quebrada Agua } \\
\text { Caliente }\end{array}$ & & $\begin{array}{l}\text { The quebrada feeds into Rio } \\
\text { Guayape, which here follows a } \\
\text { prominent NK lineament. }\end{array}$ \\
\hline & $496 / 097$ & $x$ & & & $52^{\circ}-72^{\circ} \mathrm{C}$ & \\
\hline CEDROS & $\begin{array}{l}90 / 21 \\
89 / 20 \\
94 / 20\end{array}$ & & $x$ & $\begin{array}{l}\text { Quebrada Agua Tibia, } \\
\text { Agua Tibia, and Quebrada } \\
\text { Caliente }\end{array}$ & & \\
\hline CHOLOMA & $034 / 233^{*}$ & $x$ & $x$ & $\begin{array}{l}\text { Cerro El Agua Caliente } \\
\text { and Laguna } \\
\text { Agua Caliente }\end{array}$ & $36^{\circ}-40^{\circ} \mathrm{C}$ & \\
\hline CIFUERTES & $03 / 55$ & $x$ & $x$ & $\begin{array}{l}\text { Agua Caliente and } \\
\text { Quebrada Agua Caliente }\end{array}$ & $72^{\circ}-80^{\circ} \mathrm{C}$ & $\begin{array}{l}\text { Very near boraer with Micaragua: } \\
\text { location as reported in Gislason } \\
\text { (1980) geothermal report is } \\
\text { suspect. }\end{array}$ \\
\hline \multirow[t]{3}{*}{ CIUDAD DE MACAOME } & $66 / 98$ & & $x$ & Agua Tibia & & Part of the Pavana thermal area. \\
\hline & $599 / 925$ & $x$ & & & $36^{\circ} \mathrm{C}$ & \\
\hline & $497 / 956$ & $x$ & & & $41^{\circ}-93^{\circ} \mathrm{C}$ & $\begin{array}{l}\text { Hot springs on east bank of Río } \\
\text { Nacaome. }\end{array}$ \\
\hline COLOMONCAGUA & $42 / 42$ & & $x$ & El Agua Caliente & & 、 \\
\hline \multirow[t]{2}{*}{ ComaYAGUA } & $312 / 956$ & $x$ & & & $33^{\circ}-50^{\circ} \mathrm{C}$ & \\
\hline & $233 / 887$ & $x$ & & & $32^{*}-38^{\circ} \mathrm{C}$ & $\begin{array}{l}\text { Probably associated with normal } \\
\text { faults bounding the Comayagua } \\
\text { graben on the west. }\end{array}$ \\
\hline
\end{tabular}

[An W In the coordinates column means that this location was taken from the Gislason (1980) geothermal report.] 


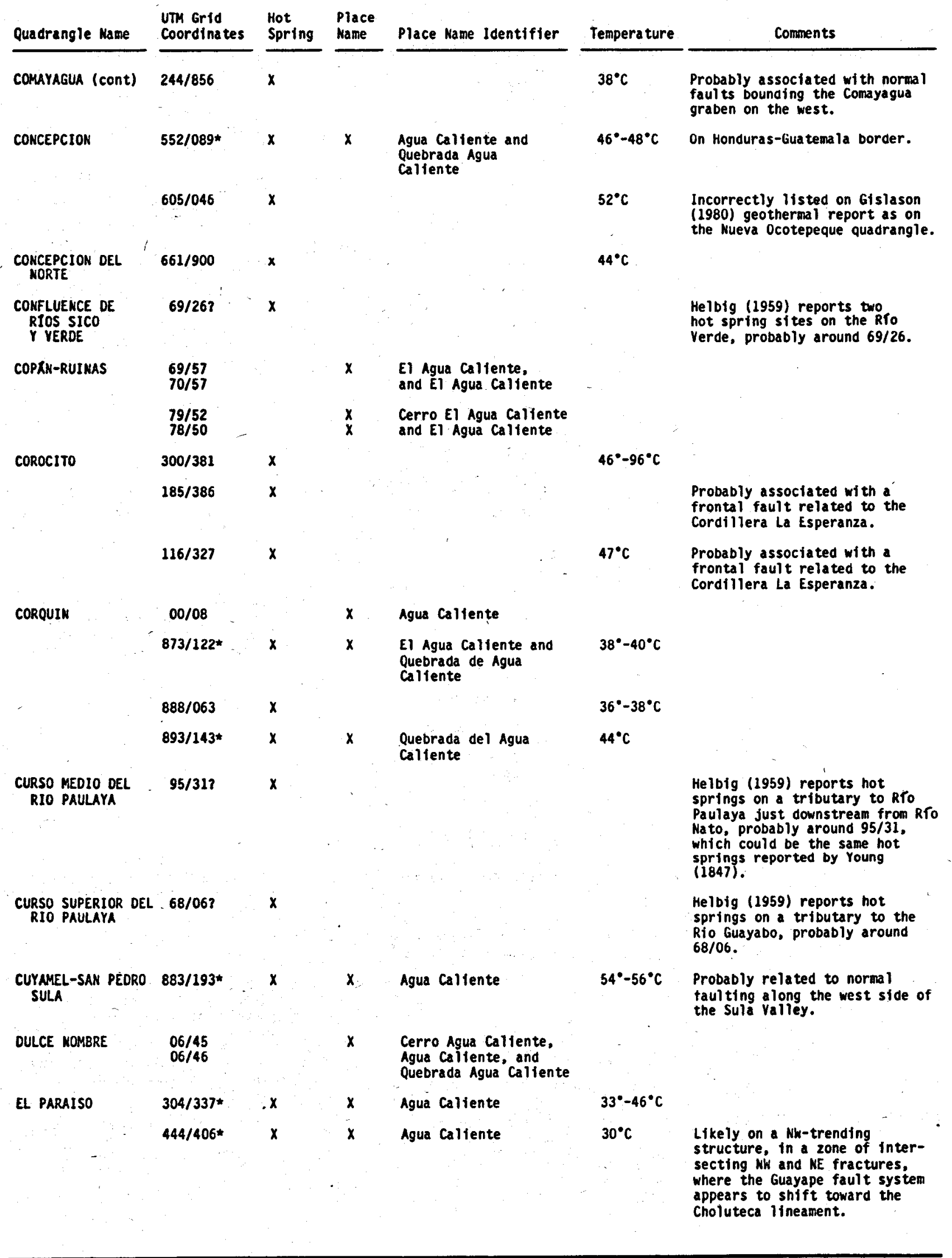


Quadrangle Name

UTM Grid Hot Place

Coordinates Spring Name

Place Mame Identifier

Temperature

Comments

EL PORTAL DEL

$19 / 92 \quad x$

IMFIERHO

$19 / 94$

$x$

E) Agua Caliente

Quebrada E1 Agua

$70^{\circ} \mathrm{C}$

Caliente

unknown

$\mathbf{x}$

$74^{\circ}-76^{\circ} \mathrm{C}$

This is probably the site naned

Hacienda Agua Caliente in

Gislason (1980): if so, the

location given in that report is inaccurate.

EL PORVENIR $92 / 24 \quad X$

$90^{\circ}-100^{\circ} \mathrm{C}$ San Ignacio thermal site, large

spring located in Caserto

Los Almendros in Gislason (1980): probably south of the hacienda Agua Caliente site.

volume thermal springs with much mineralization; probably associated with the intersection of the Arenal fault with the fault bounding the Pm block: cited in Simonson (1977) and Aldrich et al. (1986).

$11 / 57$

EL ROSARIO

224/118

X

ERANDIQUE

$58 / 79$

$64 / 82$

GRACIAS

318/098*

$x$

28/05*

$x$

$12 / 16$

$27 / 04 ?$

$94 / 72$

$00 / 69$

$00 / 70$

$00 / 7$

05/67

$05 / 84$

ILANGA

JIMIA

942/041*

$39 / 44$

$x$

496/416* $x$

$59 / 34$

LA BACADIA

$59 / 34$
$61 / 37$ $x$

El Agua Caliente

Balneario El Chimbo

$x$

$\mathbf{x}$

X

$\mathbf{X}$

$\mathbf{X}$
Quebrada El Agua Callente

Quebrada El Agua Callente

Quebrada Agua callente

Agua Callente

Quebrada Agua Caliente $40^{\circ} \mathrm{C}$

$56^{\circ} \mathrm{C}$

No thermal place name: local

inhabitants say that there are hot springs near Oromllaca:

could be on La Campa quadrangle.
Rather large volume spring feeds Dalneario. $x$ El Agua Caliente

$x \quad$ Agua Caliente, Cerro

$X$ El Agua Caliente, and

$X$ Quebrada del Agua

Caliente

$x$

$x$

$x$

X

E) Agua Calfente and Fuente de Agua Termal

Quebrada del Agua Caliente

Sabana Agua Caliente $\quad 52^{\circ} \mathrm{C}$

$52^{\circ} \mathrm{C}$

$85^{\circ}-101^{\circ} \mathrm{C}$

Sambo Creek thermal site;

probably associated with Cordillera Hombre de Dios frontal fault.

$81^{\circ}-86^{\circ} \mathrm{C}$ Springs probably associated with Cordillera Nombre de Dios frontal fault.

This stream also on adjacent San jose de Río Tinto quadrangle.
Agua Caliente and

Quebrada de Agua

Caliente
In the Aguan Valley and presumably related to the Aguan fault system. 


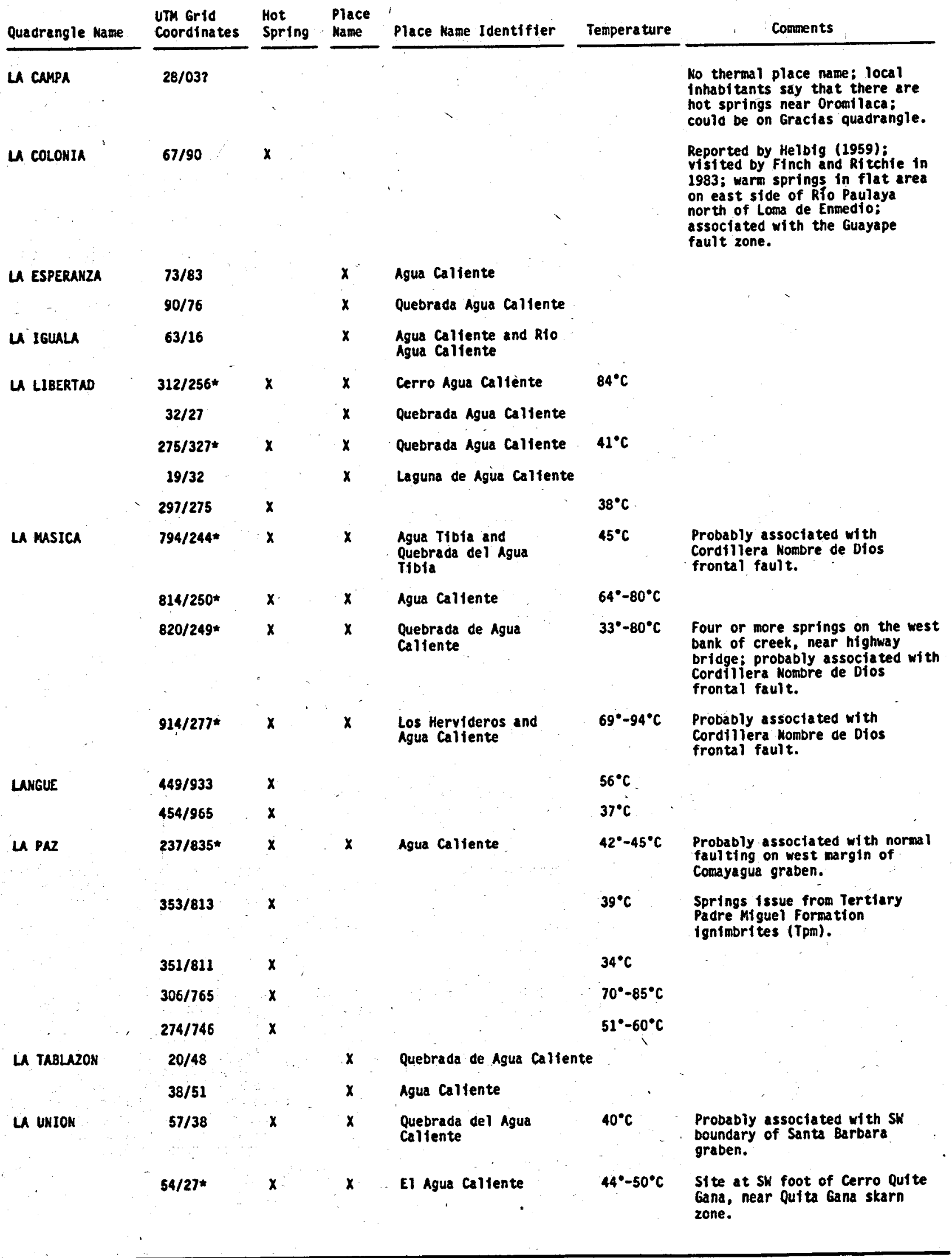




\begin{tabular}{|c|c|c|c|c|c|c|}
\hline Quadrangle Name & $\begin{array}{l}\text { UTM Grid } \\
\text { Coordinates }\end{array}$ & $\begin{array}{l}\text { Hot } \\
\text { Spring }\end{array}$ & $\begin{array}{l}\text { Place } \\
\text { Hame }\end{array}$ & Place Name Identifier & Tempera ture & Comments \\
\hline \multirow[t]{3}{*}{ LA VIRTUD } & $267 / 532 \star$ & $x$ & $x$ & $\begin{array}{l}\text { Quebrada de Agua } \\
\text { Caliente, Agua } \\
\text { Caliente, and Quebrada } \\
\text { de Agua Caliente }\end{array}$ & $66^{\circ}-74^{\circ} \mathrm{C}$ & $!$ \\
\hline & $238 / 525$ & $x$ & & & $51^{\bullet}-56^{\circ} \mathrm{C}$ & \\
\hline & $211 / 504$ & $x$ & & & $54^{\circ}-98^{\circ} \mathrm{C}$ & $\begin{array}{l}\text { On banks of Río Lempa, } \\
\text { Honduras-Ei Salvador border. }\end{array}$ \\
\hline \multirow[t]{2}{*}{ LAS FLORES } & unknown & $x$ & & & & $\begin{array}{l}\text { No thermal place name; hot } \\
\text { spring reported by University of } \\
\text { Kentucky archaeologist Ken Hirth } \\
\text { to be on Rfo Sulaco between La } \\
\text { Conce }(31 / 60) \text { and the mouth of } \\
\text { the Rio Chilistagua }(40 / 63) \text {. }\end{array}$ \\
\hline & $199 / 624$ & $x$ & & & $51^{\circ} \mathrm{C}$ & $\begin{array}{l}\text { Located imediately downstream } \\
\text { from El Cajón dam site. }\end{array}$ \\
\hline \multirow[t]{2}{*}{ LEPAERA } & $126 / 265$ & $x$ & & & $42^{\circ} \mathrm{C}$ & \\
\hline & $25 / 26$ & $x$ & & & $45^{\circ}-51^{\circ} \mathrm{C}$ & \\
\hline LEPAGUARE & $636 / 042$ & $x$ & & & $52^{\circ}-60^{\circ} \mathrm{C}$ & \\
\hline $\begin{array}{l}\text { MACUELIZO-YALLE } \\
\text { DE QUIMISTAN }\end{array}$ & $33 / 85$ & & $x$ & Agua Caliente & & $\begin{array}{l}\text { Possibly associated with the } \\
\text { Chamelecon fault zone. }\end{array}$ \\
\hline \multirow[t]{2}{*}{ мavio } & $555 / 458$ & $x$ & $x$ & $\begin{array}{l}\text { Manantial de Agua } \\
\text { Terma 1, Rfo El Agua } \\
\text { Caliente, and Agua } \\
\text { Calfente }\end{array}$ & & $\begin{array}{l}\text { Group of springs issuing from } \\
\text { Tertiary Matagaipa Formation } \\
\text { (Tm). }\end{array}$ \\
\hline & 785/484* & $x$ & $x$ & $\begin{array}{l}\text { Cerro El Agua Caliente, } \\
\text { El Agua Caliente, and } \\
\text { Rio El Agua Callente }\end{array}$ & $74^{\circ} \mathrm{C}$ & \\
\hline MARCALA & $77 / 55$ & & $x$ & Quebrada Agua Caliente & , & On possible NW lineament. \\
\hline \multirow[t]{2}{*}{$\begin{array}{l}\text { MERCEDES DE } \\
\text { ORIENTE }\end{array}$} & $98 / 37$ & & $x$ & Agua Calfente & & \\
\hline & $06 / 38$ & & $x$ & Río del Agua Caliente & & \\
\hline MEZAPA & $292 / 239$ & $x$ & & & $61^{\circ}-78^{\circ} \mathrm{C}$ & \\
\hline \multirow[t]{2}{*}{ MIMAS DE ORO } & $701 / 227 *$ & $x$ & $x$ & $\begin{array}{l}\text { Quebrada Agua Tibia and } \\
\text { town of Agua Caliente }\end{array}$ & $54^{\circ}-70^{\circ} \mathrm{C}$ & 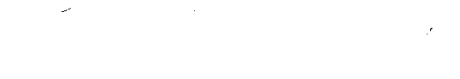 \\
\hline & $52 / 37$ & & $x$ & Quebrada Agua Tibia & & \\
\hline $\begin{array}{l}\text { MONTATK DE } \\
\text { BOTSDEROS }\end{array}$ & $80 / 11$ & & $x$ & Agua Callente & & $\begin{array}{l}\text { This place name refers to a site } \\
\text { on the adjacent Confluence de } \\
\text { Rfos Aguan-Mame quadrangle. }\end{array}$ \\
\hline \multirow[t]{2}{*}{ MONTANUELAS } & $225 / 424 *$ & $x$ & $x$ & Agua Caliente & $37^{\circ}-46^{\circ} \mathrm{C}$ & \\
\hline & $218 / 432 \star$ & & & & & - \\
\hline \multirow[t]{2}{*}{ MOROCELLI } & $080 / 533$ & $x$ & $x$ & Laguna Agua Caliente & & $\begin{array}{l}\text { A sma 11, tepid laguna at the } \\
\text { base of what is likely a fault- } \\
\text { line scarp. }\end{array}$ \\
\hline & $12 / 57$ & $x$ & $x$ & Agua Caliente & $75^{\circ} \mathrm{C}$ & $\begin{array}{l}\text { Site no. } 54 \text { on Gislasen }(1980) \\
\text { geothermal report (1n which the } \\
\text { location of this site is } \\
\text { incorrect); located on Doth } \\
\text { Danks of the Rio Choluteca; } \\
\text { issuing from Cretaceous Valle de } \\
\text { Angeles Group (Kva) beds. }\end{array}$ \\
\hline
\end{tabular}




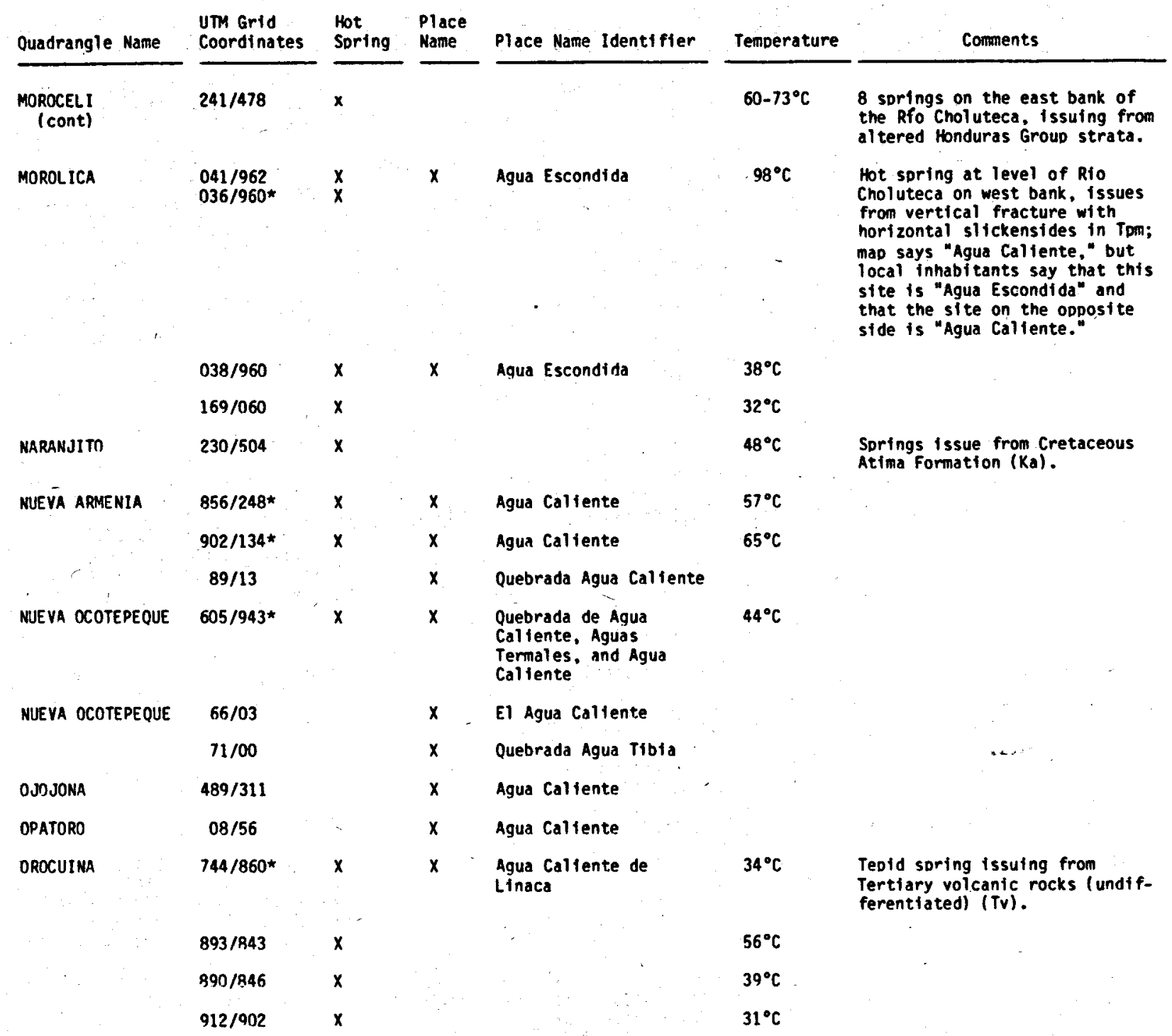

This piace name orobably came from the site at $555 / 458$ on the adjacent Manto quadrangle.

\begin{tabular}{|c|c|c|c|}
\hline PIPAERA & $\begin{array}{l}46 / 59 \\
47 / 58\end{array}$ & $\begin{array}{l}x \\
x\end{array}$ & $\begin{array}{l}\text { Quebrada El Agua } \\
\text { Caliente and EI } \\
\text { Agua Caliente }\end{array}$ \\
\hline UEBLO VIE JO & $54 / 92$ & $x$ & Quebrada Agua Calfente \\
\hline PUNTA SAL & $26 / 53$ & $x$ & Cerro Agua Callente \\
\hline
\end{tabular}

RÍ AGUÁN

$74 / 79$

$x$

Agua Caliente 


\begin{tabular}{|c|c|c|c|c|c|c|}
\hline Quadrangle Name & $\begin{array}{l}\text { UTM Grid } \\
\text { Coordinates }\end{array}$ & $\begin{array}{l}\text { Hot } \\
\text { Spring }\end{array}$ & $\begin{array}{l}\text { Place } \\
\text { Name }\end{array}$ & Place Name Identifier & Temperature & Comments \\
\hline RIO LEAN & $66 / 22$ & & $x$ & $\begin{array}{l}\text { Quebrada de Agua } \\
\text { Caliente }\end{array}$ & & $\begin{array}{l}\text { Probably associated with } \\
\text { Cordiliera Nombre de Dios } \\
\text { frontal fault. }\end{array}$ \\
\hline \multirow[t]{3}{*}{ RIO LINDO } & 977/705* & $x$ & $x$ & $\begin{array}{l}\text { Laguna Agua } \\
\text { Cailente (Aguas } \\
\text { Termales) }\end{array}$ & $50^{\circ}-75^{\circ} \mathrm{C}$ & $\begin{array}{l}\text { Warm springs associated with } \\
\text { considerable mineralization; } \\
\text { possibly associated with graben } \\
\text { faulting in the Sula Valley. }\end{array}$ \\
\hline & $04 / 75$ & & $x$ & Quebrada Agua Caliente & . & Part of the El Olivar site. \\
\hline & $060 / 733$ & $x$ & $x$ & Laguna El Playon & $35^{*}-71^{\circ} \mathrm{C}$ & $\begin{array}{l}\text { The El olivar thermal site; } \\
\text { several hot springs associated } \\
\text { with a small fault-line scarp. }\end{array}$ \\
\hline RIO NEGRO & $89 / 38$ & & $x$ & $\begin{array}{l}\text { quebrada del agua } \\
\text { Caliente }\end{array}$ & & . \\
\hline RIO TOCOA & $112 / 322$ & $x$ & & & $35^{\circ}-44^{\circ} \mathrm{C}$ & , \\
\hline SABANA GRAKDE & $72 / 16$ & & $x$ & Quebrada Agua Caliente & & - \\
\hline SABA-TOCOA & $920 / 314 *$ & $x$ & $x$ & Agua Caliente & $70^{\circ} \mathrm{C}$ & $\begin{array}{l}\text { Site in the middle of the Aguán } \\
\text { Yalley in what should be an } \\
\text { alluviated plain; presumably } \\
\text { associated with the Aguan fault } \\
\text { system. }\end{array}$ \\
\hline SAN ANDRES & $18 / 69$ & & $x$ & Agua Caliente & & \\
\hline \multirow[t]{3}{*}{$\begin{array}{l}\text { SAM ANTONIO DEL } \\
\text { MORTE }\end{array}$} & $22 / 35$ & & $x$ & Agua Caliente & . & \\
\hline & $21 / 47$ & & $x$ & Quebrada Agua Callente & & \\
\hline & $44 / 31$ & & $x$ & $\begin{array}{l}\text { Quebrada del Agua } \\
\text { Caliente }\end{array}$ & & \\
\hline SAN BUEMAYENTURA & $95 / 29$ & & $x$ & Quebrada Agua Tiola & & \\
\hline SAK ESTEBAK & $22 / 837$ & & & & & $\begin{array}{l}\text { No place name; fossil spring } \\
\text { mound, exact iocation not } \\
\text { recorded, seen by Finch beside } \\
\text { road near El ciruelo, probably } \\
\text { in grid square } 22 / 83 \text {; site is in } \\
\text { oroad alluviated valiey. }\end{array}$ \\
\hline SAN FRANCISCO & $779 / 413^{*}$ & $x$ & $x$ & $\begin{array}{l}\text { El Agua Caliente and } \\
\text { Quebrada de Agua } \\
\text { Caliente }\end{array}$ & $68^{\circ} \mathrm{C}$ & $\begin{array}{l}\text { Site appears to be in coastal } \\
\text { plain alluvium, but thermal } \\
\text { activity could be related to } \\
\text { Cordillera Nombre de Dios } \\
\text { frontal fauit. }\end{array}$ \\
\hline \multirow[t]{3}{*}{ SAN ISIDRO } & $841 / 212$ & $x$ & & & $65^{\circ} \mathrm{C}$ & $\begin{array}{l}\text { El zarzal thermal site hot } \\
\text { springs issue from ka on east } \\
\text { bank of Rio Ulua at water level } \\
\text { (inundated in wet season); cited } \\
\text { in Finch (1972). }\end{array}$ \\
\hline & $753 / 142$ & $x$ & & & $71^{\circ}-90^{\circ} \mathrm{C}$ & \\
\hline & $861 / 134$ & $x$ & & & $33^{\circ} \mathrm{C}$ & \\
\hline $\begin{array}{l}\text { SAN JOSE DE } \\
\text { COLIMAS }\end{array}$ & $56 / 61$ & & $x$ & $\begin{array}{l}\text { Quebrada de Agua } \\
\text { Caliente }\end{array}$ & & \\
\hline $\begin{array}{l}\text { SAM JOSE DE RIO } \\
\text { TINTO }\end{array}$ & $58 / 40$ & & $x$ & $\begin{array}{l}\text { Quebrada de Agua } \\
\text { Caliente }\end{array}$ & & $\begin{array}{l}\text { This place name probably relates } \\
\text { to the thermal site name on the } \\
\text { adjacent La Bacadia quadrangle. }\end{array}$ \\
\hline
\end{tabular}




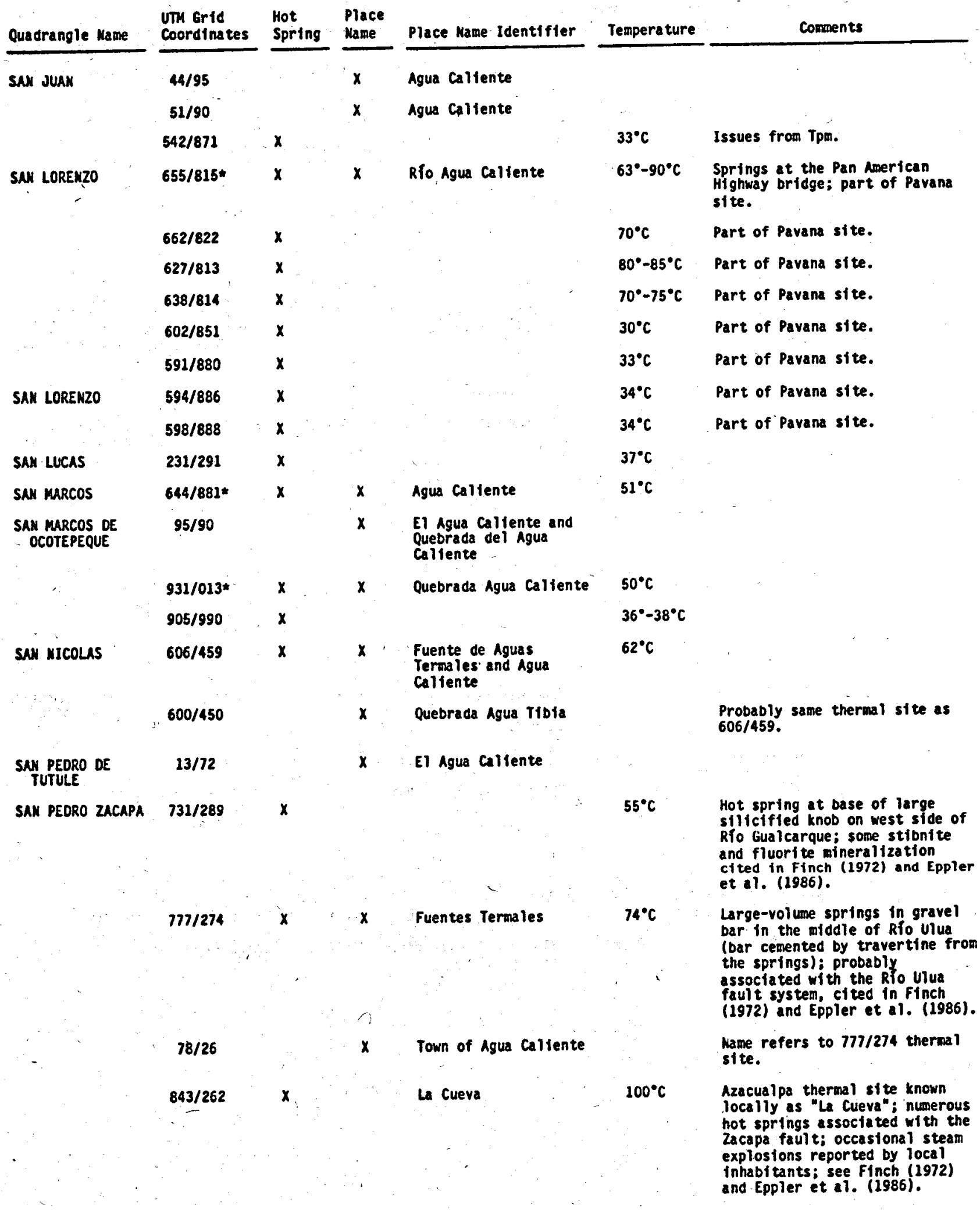




\begin{tabular}{|c|c|c|c|c|c|c|}
\hline Quadrangle Mame & $\begin{array}{l}\text { UTh Grid } \\
\text { Coordinates }\end{array}$ & $\begin{array}{l}\text { Hot } \\
\text { Spring }\end{array}$ & $\begin{array}{l}\text { Place } \\
\text { Name }\end{array}$ & Place Name Identifier & Tempera ture & Comments \\
\hline \multirow[t]{2}{*}{$\begin{array}{l}\text { SAK PEDRO ZACAPA } \\
\text { (cont) }\end{array}$} & $702 / 344$ & $x$ & & & $32^{\circ} \mathrm{C}$ & $\begin{array}{l}\text { Possibly related to Ulua fault } \\
\text { system (Finch, 1972). }\end{array}$ \\
\hline & $716 / 342$ & $x$ & & & $30^{\circ} \mathrm{C}$ & Cited in Finch (1972). \\
\hline \multirow{5}{*}{ SAMTA MARIA } & $865 / 417$ & $x$ & & & $76^{\circ}-83^{\circ} \mathrm{C}$ & \\
\hline & $878 / 397$ & $x$ & & & $56^{\circ} \mathrm{C}$ & \\
\hline & $875 / 395$ & $x$ & & & $76^{\circ} \mathrm{C}$ & \\
\hline & $990 / 403$ & $x$ & & & $74^{\circ}-84^{\circ} \mathrm{C}$ & \\
\hline & $989 / 404$ & $x$ & & & $75^{\circ}-81^{\circ} \mathrm{C}$ & \\
\hline $\begin{array}{l}\text { SANTA ROSA DE } \\
\text { COPRN }\end{array}$ & $\begin{array}{l}923 / 329 \star \\
928 / 327 \star\end{array}$ & $\begin{array}{l}x \\
x\end{array}$ & $\begin{array}{l}x \\
x\end{array}$ & $\begin{array}{l}\text { Quebrada del Agua } \\
\text { and El Agua Caliente }\end{array}$ & $79^{\circ}-101^{\circ} \mathrm{C}$ & $\begin{array}{l}\text { The Platanares thermal site; } \\
\text { numerous hot springs issuing } \\
\text { from Tpm; cited in Heiken et al. } \\
\text { (1986). }\end{array}$ \\
\hline TEGUCIGALPA & 779/634* & $x$ & $x$ & Agua Caltente. & $53^{\circ} \mathrm{C}$ & \\
\hline TEUPASENTI & $533 / 813^{*}$ & $x$ & $x$ & El Agua Caliente & $50^{\circ}-81^{\circ} \mathrm{C}$ & $\begin{array}{l}\text { Local inhabitants report that } \\
\text { water here is sulfurous smelling } \\
\text { and hot enough to boil eggs. }\end{array}$ \\
\hline TRUJILLO & $191 / 612$ & $x$ & & & $64^{\circ} \mathrm{C}$ & \\
\hline \multirow[t]{2}{*}{ VALLECILLLO } & $66 / 19$ & & $x$ & $\begin{array}{l}\text { Quebrada de Agua } \\
\text { Caliente }\end{array}$ & 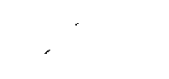 & \\
\hline & $65 / 19$ & & $x$ & $\begin{array}{l}\text { Rio Grande del Agua } \\
\text { Caliente }\end{array}$ & & $\begin{array}{l}\text { This stream flows near town of } \\
\text { Agua Caliente on adjacent Minas } \\
\text { de Oro quadrangle, and its name } \\
\text { may refer to thermal sites } \\
\text { there. }\end{array}$ \\
\hline VALLE DE MACO & $906 / 054$ & $x$ & & & $50^{\circ} \mathrm{C}$ & \\
\hline $\begin{array}{l}\text { YILLA DE SAN } \\
\text { FRANCISCO }\end{array}$ & $10 / 81$ & & $x$ & $\begin{array}{l}\text { Agua Caltente and } \\
\text { Quebrada del Agua } \\
\text { Callente }\end{array}$ & & $\begin{array}{l}\text { Located on west flank of Montaña } \\
\text { El Chile, which is a massive } \\
\text { plug-like body of Tv. }\end{array}$ \\
\hline \multirow[t]{3}{*}{ YILLARUEYA } & $09 / 82$ & & $x$ & Quebrada de Agua Caliente & & \\
\hline & $04 / 77$ & & $x$ & Quebrada Agua Caliente & & $\begin{array}{l}\text { Part of the El olivar thermal } \\
\text { site on the adjacent Río Lindo. } \\
\text { quadrangle. }\end{array}$ \\
\hline & $051 / 803$ & $x$ & & & $40^{\circ} \mathrm{C}$ & \\
\hline YORITO & $53 / 59$ & & $x$ & El Agua Caliente & & \\
\hline YUSCARAN & $246 / 314$ & $x$ & & & $80^{\circ} \mathrm{C}$ & Springs issue from Tpm. \\
\hline ZAMBRANO & $626 / 727$ & $x$ & $x$ & $\begin{array}{l}\text { Balneario San Francisco } \\
\text { hot springs }\end{array}$ & $36^{\circ} \mathrm{C}$ & Associated with fault in $\mathrm{Tpm}$. \\
\hline
\end{tabular}




\section{ACKNOWLEDGMENT}

Grateful acknowledgment is made to Ingeniero Rodney Saubers, Project Chief of the Inter-American Geodetic Survey Honduras Project, for his invaluable help, without which, this listing could not have been compiled.

\section{REFERENCES}

Aldrich, M. J., Eppler, D. B., Heiken, G. H., Flores, W., Ramos, N., and Ritchie, A. R., 1986, San Ignacio (La Tembladera) Geothermal Site, Departamento de Francisco Morazán, Honduras, Central America, Los Alamos National Laboratory report LA-10696-MS.

Eppler, D. B., Baldridge, S., Perry, F., Flores, W., Paredes, J. R., and Finch, R. C., 1986, Geology of the Azacualpa Geothermal Site, Departamento de Comayaqua, Honduras, Central America, Los Alamos National Laboratory report LA-10687-MS.

Finch, R. C., 1972, Geology of the San Pedro Zacapa Quadrangle, Honduras, Central America [unpub. Ph.D dissert.]: Univ. of Texas at Austin.

Gislason, G., 1980, Descriptions of geothermal manifestations in Honduras, 1980, unpub. report for the Empresa Nacional de Energía Electrica de Honduras and the United Nations Department of Co-operation for Technical Assistance for Development.

Heiken, G., Eppler, D., Wohletz, K., Flores, W., Ramos, N., and Ritchie, A., 1986, Geology of the Platanares Geothermal Site, Departamento de Copán, Honduras, Central America, Los Alamos National Laboratory report LA-10634-MS.

Helbig, K. M., 1959, Die Landschften von Nordost-Honduras: Gotha, Veb Hermann Haack.

Simonson, B. M., 1977, Geology of the El Porvenir Quadrangle, Honduras, Central America, unpub. report for the Instituto Geográfico Nacional de Honduras.

Young, T., 1847, Narrative of a residence on the Mosquito Shore during the years 1839, 1840, and 1841; with an Account of Truxillo, and the adjacent slands of Bonaca and Roatan, London, Smith Elder and Co. 


\section{LEGIBILITY NOTICE}

A major purpose of the Technical Information Center is to provide the broadest possible dissemination of information contained in DOE's Research and Development Reports to business, industry, the academic community, and federal, state, and local governments. Non-DOE originated information is also disseminated by the Technical Information Center to support ongoing DOE programs.

Although large portions of this report are not reproducible, it is being made available only in paper copy form to facilitate the availability of those parts of the document which are legible. Copies may be obtained from the National Technical Information Service. Authorized recipients may obtain a copy directly from the Department of Energy's Technical Information Center. 